\title{
The process of litigation for medical errors in Saudi Arabia and the United Kingdom
}

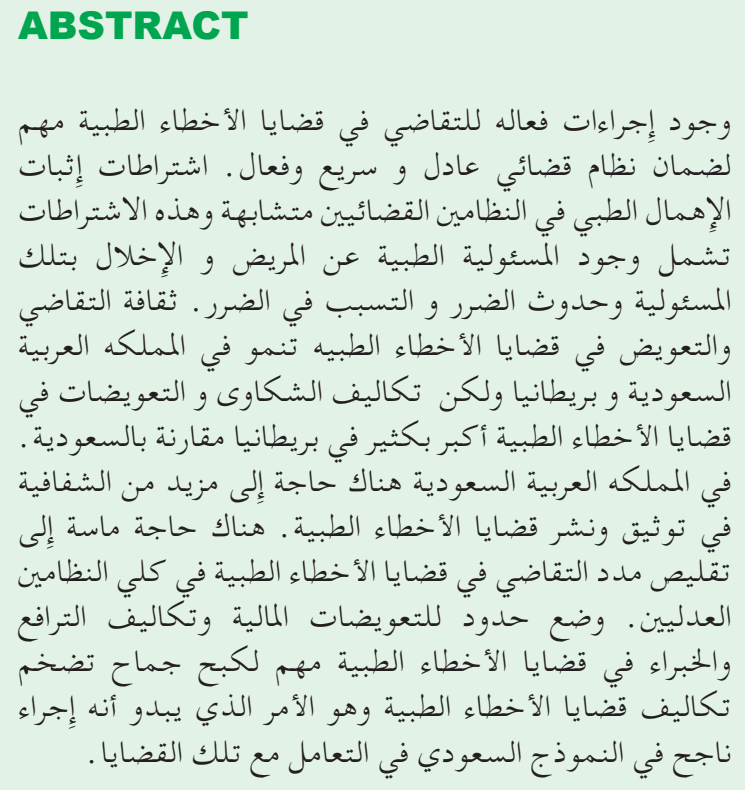

Efficient process of litigation of medical errors is key to ensure fair, speedy, and accessible justice system. The conditions for establishing medical negligence are similar in both legal systems. These conditions include the duty of care, breach of that duty of care, the damages, and establishing causation. A culture of litigation and compensation is growing in UK and Saudi Arabia; however the cost of medical claims and awarded compensations are much more in UK compared to Saudi Arabia. In Saudi Arabia, there is a need for more transparency in the documentation and publication of litigated medical errors. In addition, there is a need to introduce interventions to shorten the duration of litigation in both legal systems. Financial caps on awarded compensation and caps on expert and legal fees are potential strategies to control the cost of medical errors which seem to work well in the Saudi model.

Saudi Med J 2018; Vol. 39 (11): 1075-1081 doi: 10.15537/smj.2018.11.22854
From the Department of Family Medicine and Polyclinic, King Faisal Specialist Hospital and Research Center, Riyadh, Kingdom of Saudi Arabia.

Address correspondence and reprint request to: Dr. Abdullah $H$. Alkhenizan, Department of Family Medicine and Polyclinic, King Faisal Specialist Hospital and Research Center, Riyadh, Kingdom of Saudi Arabia.E-mail:alkhenizan@hotmail.com

ORCID ID: orcid.org/0000-0002-0269-5200

$\mathrm{M}$ edical errors are defined as the failure of a management plan to be completed as planned or the use of a wrong management plan. ${ }^{1}$ Potential medical errors can be litigated to establish clinical negligence. Clinical negligence involves a breach of the duty of care by health care professionals determined as such through the admission of the health care professional or through the appropriate legal process. In general, litigation systems aim to compensate patients who are injured because of the negligence of healthcare professionals and to prevent healthcare providers from committing medical errors as a result of clinical negligence. The process of litigation involves a series of steps taken to resolve a matter, through negotiations toward an out of court settlement, or a full court trial. The process of litigation of potential medical errors is key to establishing fair, speedy, accessible, and effective access to justice.

The purpose of this review is to compare the process of litigation of medical errors in Saudi Arabia and the United Kingdom and to draw conclusions and recommendations for both legal systems based on this comparison.

A review of the literature of the process of litigation of potential medical errors in UK and Saudi Arabia was conducted. Several literature search engines were reviewed including Medline, Embase, Heinonline, Westlaw, and Lexis Library using different key words including "litigation" "Medical Errors", "Medical Negligence", "Clinical Negligence", "compensation" "Saudi Arabia", and "United Kingdom". The bibliography of relevant papers were reviewed to retrieve 
more relevant references. The process of litigation in both systems was compared using qualitative and quantitative methods. The focus of the comparison was between claimed medical errors litigation process in Saudi Arabia and the United Kingdom. The comparison will cover the United Kingdom litigation process in general without getting into the minimal differences of the litigation procedures in England, Scotland, Wales and Northern Ireland.

The sources of legislation in the Saudi and UK legal systems are different. The legal system in Saudi Arabia is based on the Islamic jurisprudence and the Sharia law. In general, within the Saudi legal system legal precedence is not a source of legislations unlike common law in the UK legal system. In general, legal precedents in Saudi Arabia can be applied however the judge can deviate from it using his own judgement based on his own interpretation of the jurisprudence texts. The appeal court could accept or reject the judge interpretation. In Saudi Arabia courts resolutions are not publicly published and it is difficult to access; however, recently, there have been an increased trends of documenting and publishing samples of courts' resolutions.

Rates of litigations of potential medical errors in both countries are increasing. The number of new clinical claims reported by the NHS Resolution increased by $8 \%$ between 2012 and 2016. In the period 2012-2013 the reported claims were 10129 and it increased to 10965 in 2015-2016. The recently released NHS Resolution report showed a small reduction in the number of new clinical claims by $2.5 \%$ to 10,686 claims. The rates of cases reviewed by the (Sharia Medical Panels) SMPs in Saudi Arabia increased from 2002 clinical claims in 2012-2013 to 3043 in 2015-2016. ${ }^{2}$ The growth of clinical claims over the last few years is significantly more pronounced in Saudi Arabia reaching 55.2\%. This significant increase reflects the increased awareness within the community about medical errors and the rights of patients to complain and litigate health care professionals. ${ }^{3,4}$ This emphasizes the need to review, audit and improve the process of litigation in both countries, especially in Saudi Arabia.

In 2016 there was 1097 initial resolutions by the SMPs involving 2166 health care professionals (HCPs). Majority of litigated claims In Saudi Arabia involve physicians $(88.4 \%)$ and majority of convicted HCPs

Disclosure. Authors have no conflict of interests, and the work was not supported or funded by any drug company. were physicians (87.7\%). In 493 (46\%) of these initial decisions the involved health care professionals were cleared by the SMP. Sixty nine SMPs decisions (6\%) cleared the involved health care professionals through mediation or dropping of the claim. In 523 (48\%) SMPs' decisions the involved HCPs were found guilty of clinical negligence. In 352 (32\%) of SMPs decisions the involved health care professionals were found liable in the private right claim and the involved patient was compensated. ${ }^{3}$ In contrast, the rate of compensation of the involved claimants is higher in UK as $50 \%$ of claimants are compensated (including out of court settlement). ${ }^{5}$ The success rate for cases taken to court in UK was $60 \% .{ }^{6}$ Given the fact that the statistics of SMPs decisions represent a lower level of administrative decisions, the rate of compensation for claimants in Saudi Arabia could be even lower. Unfortunately, there is no published analysis of the rejected SMPs' decision; however based on a verbal communication of the head of the forensic affairs in the ministry of health, the rate of rejection of the SMPs decisions by the Court of Grievance is very low and does not exceed $10 \%$. One of the potential reasons for the higher success rate for claims in UK is the fact that more professional lawyers are involved in the process of litigation of medical errors in UK compared to Saudi Arabia. In addition, the fact that SMPs membership consists of a majority of healthcare professionals which could be a source of bias for the involved health care professionals and against patients.

In 183 of the SMPs' decisions (17\%) the involved HCPs were found guilty of public interest claims only with clearance from private rights claims. Majority of these decision are disciplinary decisions taken by the SMPs against involved healthcare professionals. On the other hand, disciplinary decisions are made in UK mainly through the MPTS. Among the 229 doctors appeared before medical practitioners tribunals in 2016, $75 \%$ of them had a sanction (of conditions, suspension or erasure) imposed on them. ${ }^{7}$ There are no statistics of pure public interest claims, however, majority of the resolutions of the SMPs related to public interest claims were raised by the SMPs during the deliberation of private rights claims which could explain the lower rates of disciplinary actions in Saudi Arabia.

A culture of compensation seems to develop in UK over the last few years. This culture is associated with increased tendency toward litigation looking for compensations for claimed medical errors. ${ }^{8}$ In the year 2016, the NHS Resolution had more than doubled the provisions arising from the different indemnity schemes it operates. The overall cost of claims increased from 
$£ 28.6$ bn in 2014-2015 to $£ 56.4$ bn in $2015-2016$. The recently published report of the NHS Resolution showed the total provisions to reach $£ 65$ billion in 2016-2017. In addition, general practitioners in UK are suffering from the increased cost and inflation of indemnity coverage. Recently, the NHS approved a plan to partially fund indemnity for general practitioners with $£ 30$ million. ${ }^{9}$ This huge overall direct cost excludes the added significant indirect cost of anxiety, distress and diverting healthcare workers from their clinical duties to the lengthy process of litigation. The significant increment in the overall cost of litigations in UK is threatening the sustainability of the provision of high quality, free healthcare services in the country. ${ }^{10}$ In UK, the number of new claims in the year 2016/17, decreased from 10,965 to 10686 , however the NHS paid more than $£ 1.08$ billion in damages with an increase of $£ 132$ million (14\%) compared to the previous year. In the year 2016/17 the amount paid out to claimant lawyers in legal fees rose by $19 \%$ to $£ 498.5$ million while defence cost increased by $5 \%$ to $£ 125.7$ million. Among the 10,686 new clinical negligence claims received by the NHS Resolution in 2016/17, 5,434 claims (50\%) were settled without payment of damages. The rate of payment per clinical claim was $£ 206207$ (989794 SAR). In Saudi Arabia the cost of clinical claims and litigations are not very well described, however based on the available data, the awarded compensations for claims related to medical errors are much lower than the awards of litigated medical errors in UK. In 2016, the SMPs in Saudi Arabia made 352 decisions that included compensations for the involved patients. About $31 \%$ of the awarded compensations were less than 10000 SAR (£2090) and about $63 \%$ of awarded compensations were less than $50,000 \operatorname{SAR}(10,400 £)$. The vast majority of awarded compensations (90.5\%) were less than 500,000 SAR $(£ 104,000)$. While compensated cases of clinical negligence in UK were awarded, on average, about 100,000 SAR ( $£ 20900)$, only 2 decisions $(0.45 \%)$ in Saudi Arabia involved compensations which exceeded 1,000,000 SAR $(208,000 £)$. The main reason for the much lower monetary compensation in Saudi Arabia is the fact that Diyah in Sharia law works as a cap for any compensation related to the wrongful death of patients as a result of medical errors. In addition, Arsh in the Sharia law sets another cap for the loss of organs or the function of organs. For example, if a patient lost the function of lower extremities because of a medical error, the maximum awarded compensation for clinical negligence in this case will be an arsh equals to, i.e. 300,000 SAR $(£ 62,500) .{ }^{11}$
The current legal system in Saudi Arabia does not provide legal aids except for major criminal offences, as indicated in Article 139 of the Judicial Procedures Law, (2013). ${ }^{12}$ Capping Diyah and Arsh compensation in addition to the lack of legal aids within the legal system in Saudi Arabia significantly limited the interest of lawyers and law firms in the business of malpractice litigations. Based on a verbal communication with the chairman of the SMPs in Riyadh, more than $90 \%$ of claimants were not represented by lawyers (they represented themselves or they were represented by a close relative). Advertisement for clinical negligence litigations is almost not existing in Saudi Arabia. On the other hand, litigations of potential medical errors represents a lucrative business in UK. The NHS Resolution paid $£ 624$ million to cover legal expenses in 2016/2017. Applying a no fault system for medical errors' injuries was rejected in UK in the Pearson Royal Commission report in 1978 and it was rejected recently, in the house of commons because it could increase the overall cost of the claims, with a reduced level of compensation per claim. ${ }^{13}$ There is an urgent need to introduce reforms to the current litigation and compensation system in UK to control the significant growth in medical errors' litigation and compensation cost.

In Saudi Arabia the government covers the cost of expert opinion within the SMPs in addition to external experts' opinion if requested through the SMPs. The government pays a specified fees for the SMPs members per meeting (600 SAR [£125]) and a similar fees for invited experts. These payment work as an additional cap which seem to be efficient in preventing the creation of a medical litigation and compensation culture in Saudi Arabia.

Cost is one of the main barriers towards Litigations against health care professionals in UK. ${ }^{6}$ However, the different legal schemes available within the UK legal system such as the "no win no fee" seem to add to the compensation and litigation culture in the country. ${ }^{8}$ There is a need to introduce reforms within the UK's legal system to control the growing culture of litigation and compensation which could include establishing caps on the awarded compensations for medical negligence claims. In addition, caps on the number and cost of experts and expert witnesses are also needed. Furthermore, reforms on the awarded compensations for loss of earnings and earning capacity should be introduced.

In general, the level of the NHS Resolution in the litigation process in UK is almost equivalent to the level of the SMPs in Saudi Arabia. In UK the mediation and conciliation process of potential medical errors starts 
before the stage of the NHS Resolution. The mediation process continues even after claims reach the NHS Resolution. In 2016/17 (67.8\%) of claims reached the NHS Resolution were resolved without formal court proceedings. About $31.5 \%$ of the claims ended up in court with less than $1 \%$ going to a full trial, with the remainder settled out of court through the established procedures of mediation. ${ }^{14}$ On the other hand, only $7 \%$ of cases which reached the SMPs in Saudi Arabia were resolved through mediation. ${ }^{3}$ The Saudi system in handling complaints of medical errors lacks a formal conciliation process. Informal mediation process probably exists within the ministry of health and the different hospital investigation committees however these efforts are not well structured or documented. In addition, the process of mediation for cases which reach the SMPs need to be transparent, structured and formal. This could be implemented through the newly established Saudi Center for Patient Safety, which was established by the ministry of health on March 2017. ${ }^{15}$

Majority of awarded cases of clinical negligence in UK come from surgical specialties including Obstetrics and Gynecology, orthopedics, and general surgery (Figure 1). ${ }^{16}$ Obstetric claims represented $10 \%$ of clinical claims by number in 2016/17; however these claims accounted for $49 \%$ of the total value of compensated claims reported in 2016/17..$^{5}$ Similar figures are seen in Saudi Arabia where doctors from surgical specialties represent the majority of compensated cases of clinical negligence lead by Obstetrics and Gynecology, general surgery, and orthopedic surgery. ${ }^{3}$ Accident and Emergency, internal medicine and pediatrics in both countries follow surgical specialties in the number of claims. The nature of these surgical specialties increases their risk of being litigated. These findings are consistent with the pattern of claims found in other countries. ${ }^{17}$ More efforts should be directed towards reducing litigations within high risk specialties. These efforts should involve analyzing the root causes of litigations in these specialties, and educating health care professionals working in these high risk specialties. In addition, more resources should be moved towards conciliation and mediation especially for cases relevant to these specialties. Analyzing and learning from litigated claims of medical errors and cases of medical negligence and developing safety guidelines from these data especially in high risk specialties such as obstetrics was shown to reduce the cost of medical liability in these specialties. ${ }^{18}$ The recently released report of the NHS Resolution included a special focus on maternity services including the analysis of claims related to maternity care and established incentives to improve the safety of maternity

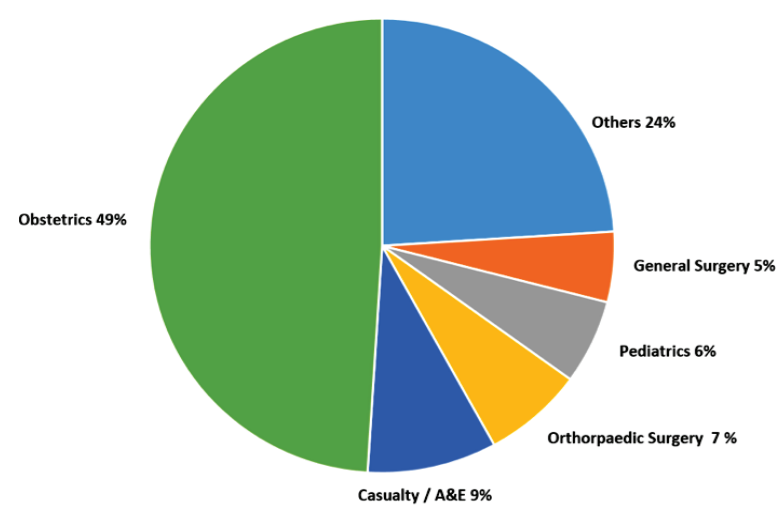

Figure 1 - Awarded compensations for clinical negligence claims per specialty in the United Kingdom (2017). ${ }^{18}$

care. ${ }^{14}$ The Saudi Center for patient safety can play a role in monitoring and analyzing medical errors at the national level, to develop appropriate proactive interventions to enhance the safety of patients in healthcare facilities.

Civil liability applies in both legal systems. The debate about the nature of the basis of medical liability whether it is contractual or torturous exists in both legal systems. Civil liability in the Saudi system is the main route of litigating private rights claims. Both legal systems apply similar elements on what constitute medical negligence including the existence of the duty of care, breach of that duty of care, the damage, and establishing causation. The role of the judge and the fact that the SMP members can't get majority voting without the judge agreeing and voting with that majority should, theoretically, ensure that health care professionals' opinion about the standards of care is logical enough for the judge to support it.

Punitive liability is used in the Saudi legal system to deal with physicians who violate the general rules and regulations of clinical practice even if no harm was inflicted on patients. Punitive liability is applied on those who practice without license, or make false advertisement about their services or fail to report reportable diseases. Punitive liability is usually proceeded under the public interest claim by the general public prosecutor. Punitive liability was referred to under section 3, articles 28-30 of the Law of Practicing Healthcare Professions (2005). The law limits the maximum momentary fine to 100,000 SAR, ( $£ 20900)$ and the maximum imprisonment of the involved health care professionals to 6 months. However the law does not prevent prosecution of the involved health care professional under other criminal court proceedings. ${ }^{19}$ The SMPs were given the authority to apply disciplinary 
liability on practitioners who violate ethical standards as indicated in Articles 31 and 32 of the Law of Practicing Healthcare Professions (2005). ${ }^{9}$ Disciplinary actions can start with a warning letter, to a fine, not exceeding 10,000 SAR $(2.700 \$)$, reaching to the revocation of the license to practice as a health care professional. The Saudi Commission for Health Specialties (SCFHS) is the regulatory body for health care professions in Saudi Arabia. The SCFHS is responsible for setting the standards for training programs in the medical field, as well as setting controls and standards for the registration of healthcare practitioners in Saudi Arabia. The SCFHS was established by Royal Decree No. M/2, (1994). ${ }^{20}$ Based on this royal decree the SCHS was not given the authority to impose disciplinary actions against health care professionals. In contrast, the Medical Practitioners Tribunal Service (MPTS) which is funded by the GMC has the main disciplinary power in UK as established in The Medical Act 1983. ${ }^{21}$ Among the 229 doctors appeared before MPTS in 2016, 75\% of them had a sanction being imposed on them. ${ }^{7}$ On the other hand, these kind of sanctions cannot be imposed on health care professionals in Saudi Arabia without going through the lengthy process of litigation as SMPs are the only authority to impose such sanctions. This made it more difficult to enforce disciplinary actions against health care professionals in Saudi Arabia.

Vicarious liability is recognized by both legal systems. Vicarious liability in cases of clinical negligence was established in UK since the case of Cassidy v Ministry of Health. ${ }^{22}$ In this case the court of appeal held that the Ministry of Health was liable for the negligence of doctors who were employed by it on contracts of service. Currently the NHS is vicariously liable for the negligent acts of all of its employees. Since 1995, the NHSLA deals with claims of potential medical errors committed by NHS trusts. The concept of vicarious liability is well established in Islamic jurisprudence. ${ }^{23}$ However, the vicarious liability within the Saudi legal system does not apply on physicians as they make their decision independent of their employers which preclude the application of the principle of vicarious liability. This was referred to in the resolution of the Court of Grievance number 151/1427. ${ }^{24}$ On the other hand, vicarious liability was applied by the SMP when patient care was claimed to be delayed because of a registration clerk. As indicated in the Court of Grievance appeal decision 341/1431 the clerk was protected with the principle of vicarious liability because the clerk was in a position where he just applies instructions of the hospital management and works under their direct supervision. ${ }^{25}$ The application of vicarious liability on non-healthcare workers and other supportive staff was not consistent by the SMP, as indicated in the Court of Grievance resolution 134/4 1427. ${ }^{26}$ This inconsistency in the SMPs decisions emphasize the need of training of members of the SMPs. In addition, codification of a law of medical negligence should be explored given the complexity of medical negligence cases and to ensure the consistency of SMPs decisions.

Malpractice insurance is mandatory for physicians in both legal systems. NHS indemnity covers all employee of NHS including physicians, nurses, and midwifes employed by the NHS. On the other hand, malpractice insurance in Saudi Arabia is optional for other health care professionals such as nurses, physiotherapists and midwifes. Medical care is usually provided by a team involving physicians, nurses and other allied health professionals. Medical negligence cases resulting from potential medical errors could involve shared responsibilities and liabilities between the healthcare team including non-health care professionals. Excluding other members of the healthcare team from malpractice insurance could put them under significant risk in case they face legal actions of potential medical errors. In addition, the Law of Practicing Healthcare Professions (2005) does not include clear consequences for not getting malpractice insurance. In contrast, in UK, there are clear consequences of not having malpractice insurance which include the suspension of the license to practice. ${ }^{27}$

Article 40 of the Law of Practicing Healthcare Professions (2005) required insured doctors and dentists to pay $5 \%$ of awarded compensation. This out of pocket payment of part of the compensation by the involved physician is generally small, but it can be a burden in cases of multiple organ damages such as cases encountered in the field of Obstetrics. In addition, in case the awarded compensation exceeded the insurance cap, the explanatory guidance required employers to pay the awarded compensation. The explanatory guidance gave employers the right to refund the paid awards from their employees. Awarded compensations especially in high risk specialties can be huge, despite the caps set by Sharia law. If a patient lost the function of multiple organs he could receive multiple diyahs as an arsh compensation. The Court of Grievance resolution (322/2 1435) upheld the decision of the SMP against one of the military hospitals to pay 5 diyahs for the loss of 5 functions of a newborn including the lower and upper extremities, fecal and urinary incontinence and mental retardation. The total compensation in that case was $1,500,000$ SAR $(£ 625,000) .{ }^{11}$ In contrast insurance schemes provided by the NHS provide full coverage for 
awarded compensations in medical negligence cases.

There are several concerns about the current malpractice policies offered by private insurance companies in Saudi Arabia. Malpractice insurance coverage is relatively limited in Saudi Arabia with a coverage which ranges between 100,000 SAR ( $£ 20900)$ to $1,000,000$ SAR $(£ 209000)$. In addition the terms and conditions of malpractice policies exclude malpractice coverage of cases related to cosmetic procedures, Acquired Immunodeficiency Syndrome (AIDs) disease and compensations related to emotional distress as indicated in the policies of several insurance companies. ${ }^{28}$ There is a need for a unified malpractice policy with wider coverage to be reviewed and approved by the Council of Cooperative Health Insurance in Saudi Arabia.

The average length of litigations in Saudi Arabia and UK is not very well documented. The litigation process seems to be longer in Saudi Arabia compared to UK. In one study $70 \%$ of litigated physicians indicated that the final verdict took two years or more. ${ }^{29}$ In another study $80 \%$ of court proceedings lasted more than 2 years. ${ }^{30}$ The average length of litigation of medical negligence cases in UK is about 18 months. ${ }^{31}$ One of the reasons of the increased length of the process of litigation in Saudi Arabia is the fact that litigation is done through the SMPs which act more like a judicial committees rather than court proceedings. The explanatory guidance of Article 40 of the Law of Practicing Healthcare Professions (2005) gave the investigators the right to apply travel ban until the resolution of the complaint which could take on average years. In one study, about $76.4 \%$ of expatriate physicians who were involved in malpractice litigations in Saudi Arabia were banned from travelling. ${ }^{29}$ Such a regulation has a significant negative impact on expatriate health care professionals involved in malpractice litigations. Expatriate health care professionals represent $80 \%$ of healthcare force in Saudi Arabia and they represent $80 \%$ of litigated health care professionals in the country. ${ }^{19}$ In general the need of such a drastic procedure is limited as malpractice insurance against medical errors is mandatory for physicians in Saudi Arabia.

In conclusion, rates of medical errors is increasing in both countries. Surgical specialties is the main source of litigated potential medical errors in both legal systems. The UK complaint management system emphasizes on the role of mediation and conciliation in the early and late stages of complaint and litigation. Mediation and conciliation is an essential part of the NHS Resolution procedures in handling claims involving potential medical errors. The Saudi system does not include formal procedures for the mediation and conciliation of potential medical errors, however mediation and conciliation are probably conducted in an informal way. A culture of litigation and compensation is growing in UK which is not yet the case in Saudi Arabia. The cost of medical claims and awarded compensations are much more in UK compared to Saudi Arabia. The growing overall cost of malpractice litigation in UK is threatening the health care system in the country. Malpractice insurance in Saudi Arabia is mandatory for dentists and physicians only. Unlike the UK, vicarious liability is applied in Saudi Arabia on allied health professionals including non-healthcare workers, but not on physicians. In general, claims of potential medical errors take longer time to reach the final verdict in Saudi Arabia compared to UK. Majority of expatriate healthcare professionals involved in litigation of potential medical errors are banned from travelling during the lengthy process of litigation in Saudi Arabia. There is a need to reform different aspects of the process of litigation of medical errors in both countries.

\section{References}

1. Kohn LT, Corrigan JM, Donaldson MS. To Err is Human: Building a Safer Health System. [Internet]. Washington (DC): National Academies Press (US); 2000 [Accessed 2000]. Available from: https://www.ncbi.nlm.nih.gov/pubmed/25077248

2. NHS Litigation Authority. NHS Litigation Authority Annual report and accounts 2015/2016. [cited 2016 July 21]. Available from URL: http://www.nhsla.com/AboutUs/ Documents/NHS_Litigation_Authority_Annual_Report_ and_Accounts_2015-2016.pdf

3. Medical Errors in Saudi Arabia. The General Directory of Forensic Centers, 2017.

4. Abdulhameed A. Revisited: The scope of medical litigations in Saudi Arabia - Analysis of closed claims over 15 years. J Health Spec 2015; 3: 162-165.

5. NHS Litigation Authority. NHS Resolution Annual report and accounts. [cited 2017]. Available from URL: http://www.nhsla. com/AboutUs/Documents/NHS\%20Resolution\%20-\%20 $\% 20$ Annual\%20report\%20and\%20accounts\%202016-17.pdf

6. Fenn P, Gray A, Rickman N, Vencappa V. Funding Clinical Negligence Cases; Access to justice at reasonable cost? London (UK): Nuffield Foundation; 2016.

7. Medical Practitioners Tribunal Service. Report to Parliament 2016. [cited 2017 July] Available from URL: https:// www.mpts-uk.org/MPTS_Report_to_Parliament_2016. pdf_71189486.pdf

8. Lord Young of Graffham. Common Sense Common Safety. [cited 2010]. Available from URL: https://www.gov.uk/ government/uploads/system/uploads/attachment_data/ file/60905/402906_CommonSense_acc.pdf

9. NHS England and the Department of Health. GP indemnity review. [cited 2016 July]. Available from URL: https://www. england.nhs.uk/wp-content/uploads/2016/07/gp-indemnityrev-summary.pdf 
10. The Medical Protection Society. The rising costs of clinical negligence: Who pays the price. [cited 2017 June]. Available from URL: https://www.medicalprotection.org/docs/defaultsource/pdfs/policy-papers/striking-a-balance-policy-paper65gs4rc7.pdf

11. Queensland Health. The Documentary of the Court of Grievance Resolution. [cited 2010 April]. Available from URL: https:// www.health.qld.gov.au/__data/assets/pdf_file/0025/164095/ qh-pol-140.pdf

12. Kemp DS. The Law of Punitive Judicial Procedures. [cited 2013]. Available from URL: https://verdict.justia.com/2013/04/08/ the-constitution-and-punitive-damages

13. Dyer C. Fault lines: resolving clinical negligence claims. $B M J$ 2013; 347: 13-14.

14. NHS Litigation Authority. NHS Resolution Business Plan 2017/18. Available from URL: http://www.nhsla.com/ AboutUs/Documents/NHS\%20Resolution\%20-\%20\%20 Business\%20Plan\%202017-18.pdf

15. Health Minister Launches Saudi Center for Patient Safety. Arab News Newspaper. 2017 March 27. Available from URL: http:// www.arabnews.com/node/1071516/saudi-arabia

16. National Audit Office. The Comptroller and Auditor General. Managing the costs of clinical negligence in trusts. National Audit Office 2017.

17. Jena AB, Seabury S, Lakdawalla D, Chandra A. Malpractice risk according to physician specialty. $N$ Engl J Med 2011; 365: 629-636.

18. Cardoso R, Zarin W, Nincic V, Adams JA, Selvaratnam I, Wilson $\mathrm{C}$, et al. Rapid scoping review of medical responses malpractice policies in obstetrics. Geneva: World Health Organization. [cited 2015]. Available from URL: http://www.who.int/health-laws/ resources/who-report_malpracticemodels_-12aug2015_final. pdf

19. Bureau of Experts at the Council of Ministers. The Law of Practicing Healthcare Professions. [cited 2005]. Available from URL: Available from URL: https://www.boe.gov.sa/ ViewSystemDetails.aspx? lang=en $\&$ SystemID $=164 \&$ Version ID $=178$
20. Cassidy v Ministry of Health. Royal Decree No. (M/2) Dated $15 / 1 / 1390 \mathrm{H}$ (corresponding to 23/3/1970). Available from URL: https://www.boe.gov.sa/printsystem.aspx?lang=ar\&syste mid $=1568$ versionid $=170$

21. The National Archives. The Medical Act 1983 (Approved Medical Practices and Conditions of Residence) and National Health Service (General Medical Services) (Scotland) Amendment Regulations 1998. [cited 1998]. Available from URL: http://www.legislation.gov.uk/cy/uksi/1998/1667/made/ data.htm?wrap=true

22. The Documentary of the Court of Grievance Resolutions. Cassidy V. Ministry of Health [1951] 2 KB 343.

23. Alzarqa M. Vicarious Liability. Islamic Jurisprudence Journal 1980; 10: 120-145.

24. The Documentary of the Court of Grievance Resolutions. Resolution Number 151/1427.

25. The Documentary of The Court of Grievance Resolutions. Resolution 341/1431.

26. The Court of Grievance Documentary Resolution 134/4 1427. Available from URL: Available at: https://www.bog.gov.sa/

27. General Medical Council. Good Medical Practice. [cited 2013]. Available from URL: https://www.gmc-uk.org/ethicalguidance/ethical-guidance-for-doctors/good-medical-practice

28. Wafa Insurance. Medical Malpractice Insurance [Internet]. [cited 2012]. Available from URL: http://www.wafainsurance. com/page-17/

29. Alkindy SA. Expatriate doctors, medical litigations, and overall patient care: Taif study. Saudi Surg Journal 2016; 4: 104-107.

30. Habib F. Obstetricians' Perception of Medico-legal Problems in Al Madinah Al Munawarah Kingdom of Saudi Arabia. Journal of Taibah University Medical Sciences 2010; 5: 66-74.

31. Quraishi NA, Hammett TC, Todd DB, Bhutta MA, Kapoor V. Malpractice litigation and the spine: the NHS perspective on 235 successful claims in England. Eur Spine J 2012; 21 : S196-S199. 